${ }^{1}$ Academic Urology Unit, Institute of Applied Health Sciences, College of Life Sciences and Medicine, University of Aberdeen, Health Sciences Building, Aberdeen AB25 2ZD

${ }^{2}$ Health Services Research Unit, Institute of Applied Health Sciences, College of Life Sciences and Medicine, University of Aberdeen

Correspondence to: $\mathrm{G}$ Nabi g. nabi@abdn.ac.uk

doi: 10.1136/bmj.39119.595081.55

\title{
Outcomes of stenting after uncomplicated ureteroscopy: systematic review and meta-analysis
}

\author{
Ghulam Nabi, clinical lecturer, J Cook, statistician, ${ }^{2}$ J N'Dow, professor of urology, ${ }^{1}$ S McClinton, consultant \\ urological surgeon ${ }^{1}$
}

\section{ABSTRACT}

Objective To investigate the potential beneficial and adverse effects of routine ureteric stent placement after ureteroscopy.

Design Systematic review and meta-analysis of randomised controlled trials.

Data sources Cochrane controlled trials register (2006 issue 2), Embase, and Medline (1966 to 31 March 2006), without language restrictions.

Review methods We included all randomised controlled trials that reported various outcomes with or without stenting after ureteroscopy. Two reviewers independently extracted data and assessed quality. Meta-analyses used both fixed and random effects models with dichotomous data reported as relative risk and continuous data as a weighted mean difference with $95 \%$ confidence intervals. Results Nine randomised controlled trials (reporting 831 participants) were identified. The incidence of lower urinary tract symptoms was significantly higher in participants who had a stent inserted (relative risk 2.25, $95 \%$ confidence interval 1.14 to 4.43 , for dysuria; 2.00 , 1.11 to 3.62 , for frequency or urgency) after ureteroscopy. There was no significant difference in postoperative requirement for analgesia, urinary tract infections, stone free rate, and ureteric strictures in the two groups.

Because of marked heterogeneity, formal pooling of data was not possible for some outcomes such as flank pain. A pooled analysis showed a reduced likelihood of unplanned medical visits or admission to hospital in the group with stents $(0.53,0.17$ to 1.60$)$, although this difference was not significant. None of the trials reported on health related quality of life. Cost reported in three randomised controlled trials favoured the group without stents. The overall quality of trials was poor and reporting of outcomes inconsistent.

Conclusions Patients with stents after ureteroscopy have significantly higher morbidity in the form of irritative lower urinary symptoms with no influence on stone free rate, rate of urinary tract infection, requirement for analgesia, or long term ureteric stricture formation. Because of the marked heterogeneity and poor quality of reporting of the included trials, the place of stenting in the management of patients after uncomplicated ureteroscopy remains unclear.

\section{INTRODUCTION}

The surgical management of ureteric stones has changed over the past few decades because of advances in instruments and techniques. ${ }^{1}$ Extracorporeal shock wave lithotripsy (ESWL) and ureteroscopy are currently the most common treatment options in clinical practice. The routine placement of ureteric stents after fragmentation and retrieval of ureteroscopic stones is questionable. ${ }^{\mathrm{w} 1 \mathrm{w} 2}$ The main advantages of stenting are the prevention of ureteric obstruction and renal pain that may develop as a result of ureteric oedema from balloon dilation or stone manipulation during ureteroscopy. Ureteric stents may aid in the passage of residual stone fragments secondary to the passive ureteric dilation that occurs with an indwelling ureteric stent ${ }^{2}$ and may prevent delayed formation of ureteric stricture. Routine placement of ureteric stents, however, is accompanied by recognised potentially troublesome urinary symptoms or morbidity, or both. ${ }^{34}$ Related complications such as migration, infection, pyelonephritis, breakage, encrustation, and stone formation are not uncommon. ${ }^{5}$ Placement of ureteric stents results in additional costs. Furthermore, unless a pull string is routinely used at the distal end of the stent, secondary cystoscopy is required to remove the stent, which has cost implications and the potential to add to the disruption of patients' lives.

Ureteroscopy is now performed with small calibre endoscopes and better intracorporeal lithotripsy devices such as holmium laser so that most patients can be treated without ureteric dilation. As a result, the need for a postprocedural stent remains questionable.

We determined the evidence that outcome with routine ureteric stent placement after uncomplicated ureteroscopy is inferior to that without stent placement.

\section{METHODS}

\section{Search strategy}

We obtained relevant trials from the Cochrane renal group's specialised register of randomised controlled trials; the Cochrane central register of controlled trials 2006; Medline and PreMedline (1966 to 31 January 2006); Embase (1980 to 31 January 2006); reference lists of urology textbooks, review articles, and relevant trials; and abstracts of conference proceedings. 
To be included randomised controlled trials had to compare stenting with no stenting after uncomplicated ureteroscopy in adults with a clinical diagnosis of ureteric stone who required intervention or who were undergoing diagnostic or therapeutic ureteroscopy for upper tract transitional cell carcinoma and had at least one of the predetermined outcomes of interest.

\section{Outcome measures}

Outcomes of interest were pain rated by patients on a validated scale, need for analgesia, lower urinary tract symptoms, unplanned medical visits or admission to hospital, complications related to the stent (such as migration, encrustation, fragmentation, ureteric erosion, and fistulas), return to normal physical activities, participants' satisfaction, health economics and health related quality of life. Trials reporting on pain were classified into two groups: those that reported pain score within or at three days or those that reported pain at or after day seven after the procedure.

\section{Quality assessment and data abstraction}

Two reviewers (GN, SMcC) independently assessed study quality using the checklist developed for the Cochrane renal group. ${ }^{6}$ Discrepancies were resolved by discussion and arbitration by a third party if necessary. They assessed concealment of allocation, intention to treat analysis, completeness of follow-up, and blinding of investigators, participants, and outcome assessors.

They screened identified titles and abstracts independently. Potentially relevant trials were retained and the full text examined. The reviewers independently extracted data. When important data were not reported, we tried to contact the authors.

\section{Study characteristics and quantitative data synthesis}

Whenever possible, we classified the studies by size and site of stones and type of ureteroscope and intracorporeal lithotripsy device used. When two or more studies reported on the same outcome we quantitatively combined results. We calculated relative risks for dichotomous data and weighted mean differences, with $95 \%$ confidence intervals, for continuous data. A fixed effects model (Mantel-Haenszel) was used unless there was evidence of substantial statistical heterogeneity, in which case we used the DerSimonian and Laird random effects model. Statistical heterogeneity of treatment effects between studies was formally tested with Cochran's test for heterogeneity $(\mathrm{P}<0.1)$. The $I^{2}$ statistic was also examined. ${ }^{7} \mathrm{We}$ explored possible sources of heterogeneity (participants, treatments, study quality). When we could not combine data quantitatively they were assessed qualitatively. All metaanalyses were performed using RevMan software (version 4.2.8).

\section{RESULTS}

Of 34 potentially relevant studies, we excluded 24 after reviewing abstracts (fig 1). We retrieved 10 articles for

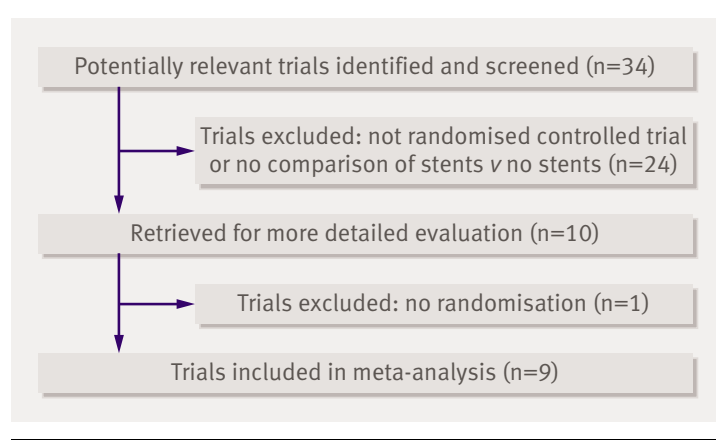

Fig 1 | Flow of studies in the systematic review

more detailed evaluation and excluded one that did not meet our inclusion criteria, ${ }^{8}$ leaving nine trials for the review. ${ }^{\text {w1-w9 }}$

\section{Study characteristics}

The nine trials were conducted in eight countries, reported on 831 participants, and were published in 2001-4 (table 1). The reported length of hospital stay after ureteroscopy varied from a few hours as an outpatient $^{\mathrm{w1} \text { w4 }}$ to two to three days. ${ }^{\mathrm{w} 7 \mathrm{w} 9}$ In two trials hospital stay varied on a case-to-case basis, ${ }^{\text {w3 }}{ }^{\text {w6 }}$ but ureteroscopy was mostly an outpatient procedure with a few patients requiring overnight hospital stay. Two trials reported hospital stays of one day. ${ }^{\text {w2 w5 }}$ One trial did not report on postoperative hospital stay. ${ }^{\text {w8 }}$

Seven studies included participants irrespective of the site of stones in the ureter ${ }^{\mathrm{w} 1}$ w2 w4-w8 and a few selectively recruited participants with stones in the lower ureter. $^{\text {w3 }}$ 9 There was no significant difference in stone size between the groups that did or did not receive a stent. Participants with intraoperative ureteric perforation or any other complications that otherwise would have required postprocedural placement of a ureteric stent were specifically excluded from the trials. One trial included participants after diagnostic or therapeutic ureteroscopy for transitional cell carcinoma of ureter or pelvicalyceal system. ${ }^{\mathrm{w} 4}$

One trial mentioned the material of the ureteric stents used. ${ }^{\text {w2 }}$ Size of stents used varied from 6 French gauge $^{\mathrm{w} 2 \mathrm{w} 3 \mathrm{w} 4 \mathrm{w6} \text { w9 }}$ to 7 French gauge, ${ }^{\mathrm{w} 5 \mathrm{w} 7}$ but two studies did not specify size. ${ }^{\text {w1 } 8}$ All trials except two used semirigid or rigid ureteroscopes ranging in size from 6.0 to 9.5 French gauge. One trial routinely used a 7.4 French gauge flexible ureteroscope for upper ureteric stones ${ }^{\mathrm{w1}}$ while another did so occasionally. ${ }^{\mathrm{w} 4}$ Two studies carried out ureteral dilatation in both groups before the introduction of the ureteroscope. ${ }^{\text {w3 }}$ w9 One study excluded participants who required dilatation. ${ }^{\mathrm{w} 4}$ In three trials ureteral dilatation to facilitate ureteroscopy was not required. ${ }^{\text {w5 } 6 \text { w8 }}$ Different sources of intracorporeal lithotripsy were used for fragmentation of large calculi, including holmium:YAG (yttrium-aluminium-garnet) laser, ${ }^{\mathrm{w} 1}$ w3 w4 w6 electromechanical, ${ }^{\text {w7 }}$ w9 electrohydraulic, ${ }^{\text {w5 }}$ and ultrasonic lithotrite. ${ }^{\text {w8 }}$

Most of the included trials failed to meet our quality criteria because of lack of information rather than 
Table1| Characteristics of included studies comparing stenting (intervention) versus no stenting (control) after uncomplicated ureteroscopy

\begin{tabular}{|c|c|c|c|c|}
\hline Study & $\begin{array}{l}\text { Interven- } \\
\text { tion/control }\end{array}$ & $\begin{array}{l}\text { Stone } \\
\text { location }\end{array}$ & Outcomes & Notes \\
\hline $\begin{array}{l}\text { Borboroglu w3 } \\
\text { USA }\end{array}$ & $53 / 60$ & Distal & $\begin{array}{l}\text { Pain scores preoperatively } \\
\text { and postoperatively at } 48\end{array}$ & $\begin{array}{l}\text { Day care procedure, except } 10 \\
\text { without and } 7 \text { with stents. }\end{array}$ \\
\hline
\end{tabular}
hours and 1 and 4 weeks, symptoms on $0-100$ scale. Analgesia requirement. Excretory urography, CT, or ultrasound at 4 weeks

Ureteroscopes 6.0 to 9.5

French gauge. Multicentre, stents removed at 3-10 days. Holmium:Yag laser used for fragmentation

\begin{tabular}{lll}
\hline Byrne $^{\mathrm{w} 4}$ USA $\quad 38 / 22$ & Variable & $\begin{array}{l}\text { Pain and lower urinary tract } \\
\text { symptoms by questionnaire } \\
\text { on postoperative days } 0,1,\end{array}$
\end{tabular}
and 6

\begin{tabular}{lll}
\hline Chen $^{\text {w5 }}$ Taiwan $\quad 30 / 30 \quad$ Variable $\quad \begin{array}{l}\text { Pain on scale of } 0-10 \text { before } \\
\text { and after operation, lower } \\
\text { urinary tract symptoms, }\end{array}$
\end{tabular}

unplanned medical visits, pyuria, KUB radiography and renograms before and 3, 7 , and 28 days after operation. Unplanned visits, return to normal activity

\begin{tabular}{|c|c|c|c|c|}
\hline $\begin{array}{l}\text { Cheungw6 }^{\text {w6 }} \\
\text { Hong Kong }\end{array}$ & $29 / 29$ & Variable & $\begin{array}{l}\text { Pain scores on scale of } 0-10 \text {, } \\
\text { symptoms and UTIs, excretory } \\
\text { urography at } 3 \text { months, } \\
\text { renogram if required for } \\
\text { ureteric strictures }\end{array}$ & $\begin{array}{l}\text { Ureteral trauma and oedema } \\
\text { assessed intraoperatively on a } \\
\text { scale of } 0-2 \text {. Participants with } \\
\text { perforation excluded from } \\
\text { study. Sample size calculated }\end{array}$ \\
\hline
\end{tabular}

Damianow2 $^{\text {w2 }} \quad$ 52/52 Variable Pain scores on scale of 0-10; Ureteral trauma and oedema
Italy lower urinary tract symptoms assessed intraoperatively on and urinary tract infections, scale of $0-2$. All participants KUB radiography. Ultrasound stayed overnight. Antibiotics in immediate postoperative used preoperatively and for period and 1 and 3 months. 5-7 days postoperatively. Excretory urography at Pneumatic lithotripsy for 6 months fragmentation. Ureteroscopes of 8.9 French gauge

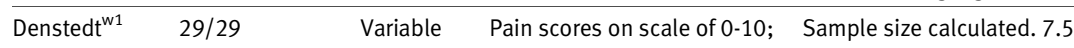
Canada analgesia requirement, lower French gauge flexible urinary tract symptoms, UTIs ureteroscopy used for upper assessed at 1,6 , and 12 weeks. KUB radiography at visit. Mainly holmium:YAG each postoperative visit and laser used for fragmentation ultrasound at 3 months

\begin{tabular}{llll}
\hline Jeong ${ }^{\text {w7 }}$ Korea $23 / 22$ & Variable & Pain and lower urinary tract & Participants admitted to \\
& symptoms assessed as mild, hospital for 2-3 days, \\
& moderate, and severe & electromechanical \\
& depending on duration and & fragmentation, 7 French gauge \\
& requirement of medications. & stents used \\
& UTls and KUB radiography at 7 \\
& and 28 days
\end{tabular}

Netto ${ }^{\text {w8 }}$ Brazil $\quad 133 / 162 \quad$ Variable Pain and postoperative
analgesia requirement, lower urinary tract symptoms on modified international prostatic symptoms score, plain radiography at immediate postoperative period and ultrasound at 2-3 months

\begin{tabular}{|c|c|c|c|c|}
\hline $\begin{array}{l}\text { Srivastavaw9 } \\
\text { India }\end{array}$ & $26 / 22$ & Distal ureter & $\begin{array}{l}\text { Operative time, postoperative } \\
\text { pain score, analgesic } \\
\text { requirement, symptoms } \\
\text { related to stent, risk of ureteral } \\
\text { stricture formation at } \\
3 \text { months }\end{array}$ & $\begin{array}{l}\text { Pneumatic lithotripsy for } \\
\text { fragmentation. Distal ureteric } \\
\text { stones treated. } 8.5 \text { semirigid } \\
\text { ureteroscopes used }\end{array}$ \\
\hline
\end{tabular}

$\mathrm{CT}=$ computed tomography; UTI=urinary tract infection; KUB=kidney, ureter, and bladder.

explicit reporting of methods that did not conform to the criteria. The trial designs were heterogeneous with regard to ureteroscope sizes, intracorporeal lithotripsy devices, postoperative analgesia, and outcome assessment and reporting. Only one trial reported on blinding (table 2).

\section{Patients' outcomes}

Patients' pain scores-Five trials measured pain scores at variable intervals after the procedure: at day one, ${ }^{\text {w9 }}$ days one and three, ${ }^{\mathrm{w} 6}$ only at day three, ${ }^{\mathrm{w} 5}$ at days three, seven, and $15,{ }^{\text {w2 }}$ and at one, six, and 12 weeks. ${ }^{\mathrm{w} 1}$ They all used a $10 \mathrm{~cm}$ visual analogue scale with 0 representing no pain and 10 representing severe pain. Experimental studies in minipigs have reported persistent mechanical ureteric oedema and urinary tract obstruction up to 96 hours after dilatation, ${ }^{9}$ suggesting that the cause of flank pain in the first three days after ureteroscopy is multifactorial and not caused by stents alone. Two trials reported no significant difference in pain scores within three days of the procedure between the groups, ${ }^{\text {w5 w9 }}$ whereas one trial favoured those without stents within three days of the procedure. ${ }^{\mathrm{w} 2}$ There was evidence of substantial heterogeneity between studies for pain scores between both immediate (within or at three days) and delayed (seven days) postoperative periods. Because of the large degree of heterogeneity we could not pool data. One trial reported a significantly higher pain score at four weeks in those with stents, ${ }^{\mathrm{w} 3}$ whereas two trials reported no significant difference at two and 12 weeks after the procedure. ${ }^{\text {w1 }}{ }^{\text {w2 }}$ In two studies the reported data were not suitable for inclusion in the meta-analysis, ${ }^{\text {w3 }}{ }^{\text {w }}$ with both trials reporting a higher pain score in participants with stents. In one other trial the method of pain measurement was not clear, ${ }^{\text {w7 }}$ though it reported no significant difference in pain perception between groups.

Requirement of analgesia - Four trials reported on the requirement for analgesia after ureteroscopic removal of ureteric stones. ${ }^{\text {w1 w6-w8 }}$ None of these studies found any significant difference in the proportion of participants who required analgesia after ureteroscopy with or without stents. Only two trials gave data suitable for meta-analysis. ${ }^{\mathrm{w} 4 \mathrm{w} 7}$ There was no difference in use of analgesics between the two groups (relative risk 1.03, $95 \%$ confidence interval 0.73 to 1.47 ).

Lower urinary tract symptoms - Eight trials reported on lower urinary tract symptoms at various lengths of follow-up. ${ }^{\text {w1-w7 w9 }}$ Combined analysis of four of these studies that reported urinary frequency or urgency showed a higher rate in participants with stents (2.00, 1.11 to 3.62 ; fig 2 ). There was also a higher rate of haematuria $(2.18,0.72$ to 6.61$)$ and dysuria $(2.25,1.14$ to 4.43) in those with stents. Data from three trials, which could not be used for meta-analysis, also showed higher rates of lower urinary tract symptoms in those

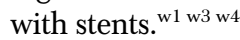

Urinary tract infections -Three trials (210 participants) reported on urinary tract infections. ${ }^{\text {w2 w6 w9 }}$ The pooled analysis showed no significant difference between the two groups $(1.09,0.48$ to 2.47 ; fig 3$)$. One trial reported significantly more pyuria in the initial postoperative period in those with stents. This 


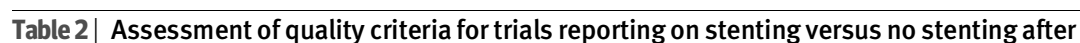
ureteroscopic retrieval of stones

\begin{tabular}{|c|c|c|c|c|}
\hline Study & $\begin{array}{c}\text { Allocation } \\
\text { concealment }\end{array}$ & Intention to treat analysis & $\begin{array}{l}\text { Completeness } \\
\text { of follow-up }\end{array}$ & Blinding* \\
\hline Borboroglu $^{\text {w3 }}$ & Adequate & $\begin{array}{l}\text { Six participants randomised to group } \\
\text { without stents removed from protocol and } \\
\text { analysis because of intraoperative injury }\end{array}$ & Reported & $\mathrm{N} / \mathrm{A}$ \\
\hline Byrne $^{\text {w4 }}$ & $\mathrm{N} / \mathrm{A}$ & $\mathrm{N} / \mathrm{A}$ & Reported & $\mathrm{N} / \mathrm{A}$ \\
\hline Chen $^{\text {w5 }}$ & Adequate & $\mathrm{N} / \mathrm{A}$ & Reported & $\mathrm{N} / \mathrm{A}$ \\
\hline Cheungw6 $^{\text {w6 }}$ & Inadequate & $\mathrm{N} / \mathrm{A}$ & Reported & $\mathrm{N} / \mathrm{A}$ \\
\hline Damianow2 $^{\text {w2 }}$ & Adequate & $\mathrm{N} / \mathrm{A}$ & Reported & $\mathrm{N} / \mathrm{A}$ \\
\hline Denstedt $^{\mathrm{w} 1}$ & Adequate & $\begin{array}{l}13 \text { participants excluded from study } \\
\text { because of intraoperative balloon dilatation }\end{array}$ & Reported & $\mathrm{N} / \mathrm{A}$ \\
\hline Jeongw7 & $\mathrm{N} / \mathrm{A}$ & $\mathrm{N} / \mathrm{A}$ & Reported & $\mathrm{N} / \mathrm{A}$ \\
\hline Netto $^{\text {w8 }}$ & $\mathrm{N} / \mathrm{A}$ & $\mathrm{N} / \mathrm{A}$ & Reported & Surgeon \\
\hline Srivastava $^{\text {w9 }}$ & Adequate & $\mathrm{N} / \mathrm{A}$ & Reported & $\mathrm{N} / \mathrm{A}$ \\
\hline
\end{tabular}

$\mathrm{N} / \mathrm{A}=$ not available (insufficient information provided).

*None of the trials provided sufficient information to determine blinding of participants or outcome assessors.

resolved, however, and there was no significant difference between groups by day 28 . $^{\text {5 }}$

Unplanned medical visits and admission to hospital-Of the participants in seven trials, 7\% (34/483) required unplanned medical visits or admission to hospital. ${ }^{4 \text { w2-w6 w9 }}$ Most participants were managed conservatively, except in three trials in which $9 / 134$ patients required another stent. ${ }^{\text {w2-w4 }}$ The pooled analysis showed a reduced likelihood of unplanned medical visits or admissions to hospital in the patients with a stent $(0.53,0.17$ to 1.60 ; fig 4$)$. One of the studies reported a significantly higher rate of unplanned admission in the patients without stents. ${ }^{\mathrm{w} 2}$ This was attributed to the use of a pneumatic intracorporeal lithotripsy device used for the fragmentation of stones, which has been shown to leave larger residual fragments compared with the holmium:YAG laser ${ }^{10}$ used in other studies. The only other trial that used similar sources of energy for intracorporeal lithotripsy did not report a significant difference in the two groups of participants. ${ }^{\mathrm{w} 9}$ Because of the large clinical heterogeneity in the use of intracorporeal lithotripsy devices between trials we could not do a pooled analysis to assess the effect of energy sources.

Return to physical activity - One trial reported no significant difference in the reported return to normal physical activities between the groups $(25(83 \%) v 24$ $(80 \%)$ at day one after the procedure). ${ }^{\text {w5 }}$

\section{Efficacy outcomes}

Ureteric strictures/stone free rate - Of the nine trials, six reported on the rate of ureteric stricture formation. ${ }^{\text {w1-w3 } w 6 \text { w8 }}$ 99 There was no difference in the

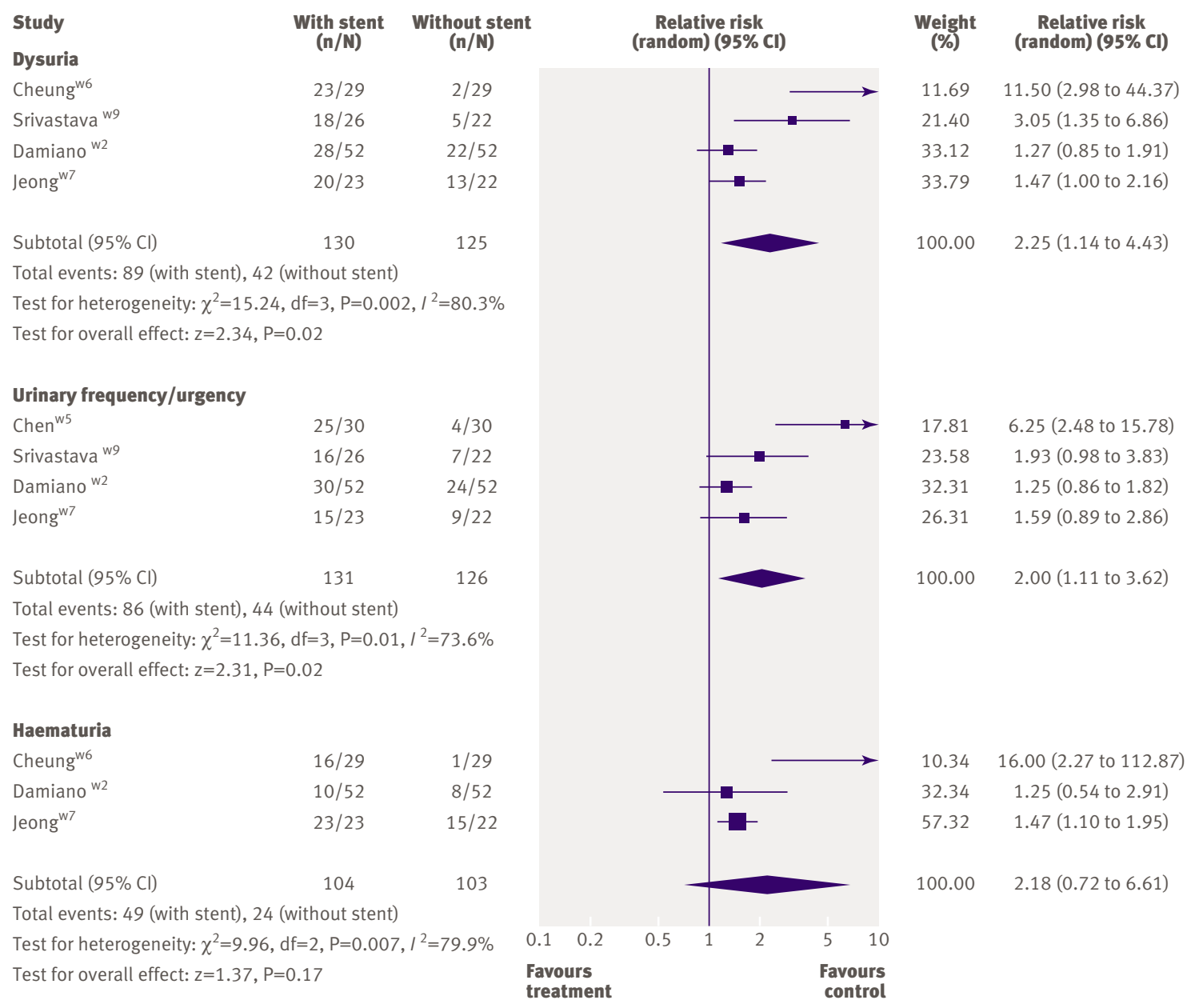




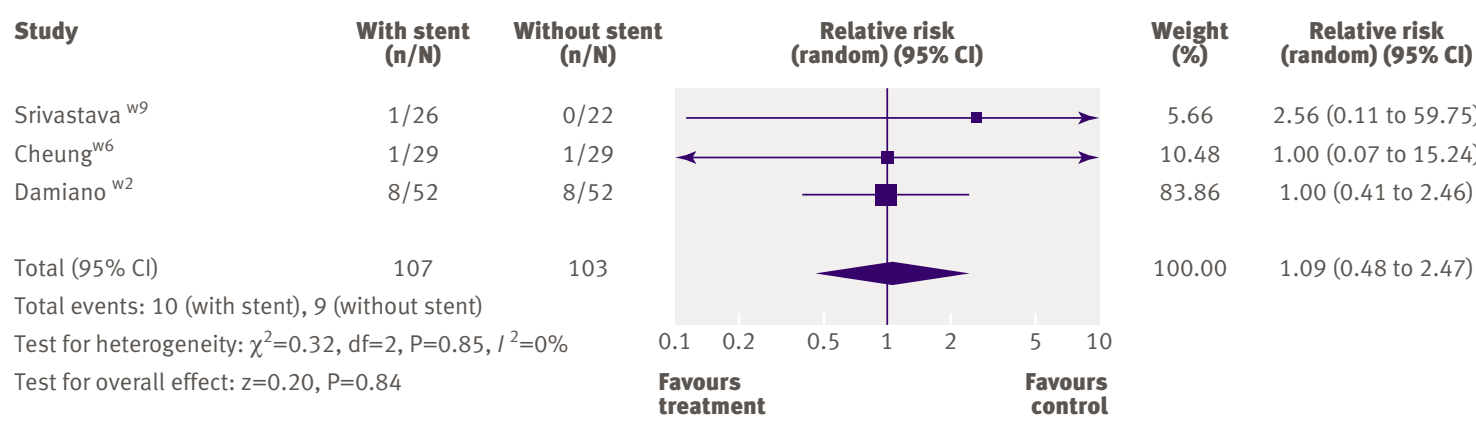

Fig 3 | Urinary tract infections proved by culture in patients with and without stents after ureterosopy

proportion of participants developing strictures with or without stents. Similarly, none of the trials reported significant differences in the stone free rates between participants with or without a stent.

Health related quality of life

None of the trials reported on the quality of life of participants. One of the trials assessed participants' preference by asking those who received a stent whether they would prefer to undergo ureteroscopy without placement of a stent if they needed one in the future. ${ }^{\text {w9 }}$ Around two thirds of participants in the stented group said they would prefer not to have stents after any future ureteroscopy.

\section{Health economics}

Three studies reported on the cost per patient with or ${\text { without a stent. }{ }^{\mathrm{w} 4} \mathrm{w} 8 \mathrm{w} 9}$ Though costs were higher for the group with stents in all the three trials, the methods used to estimate costs were not well described and it is unclear how appropriate any of the costs estimates were and whether any were transferable to other settings. In one trial, the reported costs per patient were based on hospital charges but had been incorrectly calculated. ${ }^{\mathrm{w} 8}$ The operation time (minutes), a key cost driver, was consistently longer in the group with stents (weighted mean difference $5.37,95 \%$ confidence interval 2.37 to $8.36, I^{2}=0$, fig 5). ${ }^{\text {w1-w6 w9 }}$

\section{DISCUSSION}

\section{Principal findings}

In this systematic review we found that stenting after ureteroscopy is associated with increased lower urinary tract symptoms such as dysuria and frequency or urgency. We evaluated the benefits, harms, and costs of stenting after uncomplicated ureteroscopy, most commonly for the management of ureteric stones. Unplanned medical visits and admissions to hospital were more common in the group without stents, though the differences were not significant. The clinical implication of these findings needs further research as the present level of evidence is based on trials with marked clinical heterogeneity because of the use of different sizes of ureteroscopes, different intracorporeal lithotripsy devices, and variation in practice and experience. Moreover, the definition of "uncomplicated ureteroscopy" varied between studies. We could not ascertain whether there was a difference in postoperative pain because of the small number of studies reporting on this and the varied results.

We found no significant difference between the groups with and without stents in the need for postprocedural analgesia, urinary tract infection, stone clearance rates, and ureteric stricture development. These outcomes, however, were not reported consistently across the studies. None of the trials reported on health related quality of life. No trials investigated the impact of stent design and material on outcome, especially as related to quality of life. In a randomised study Joshi et

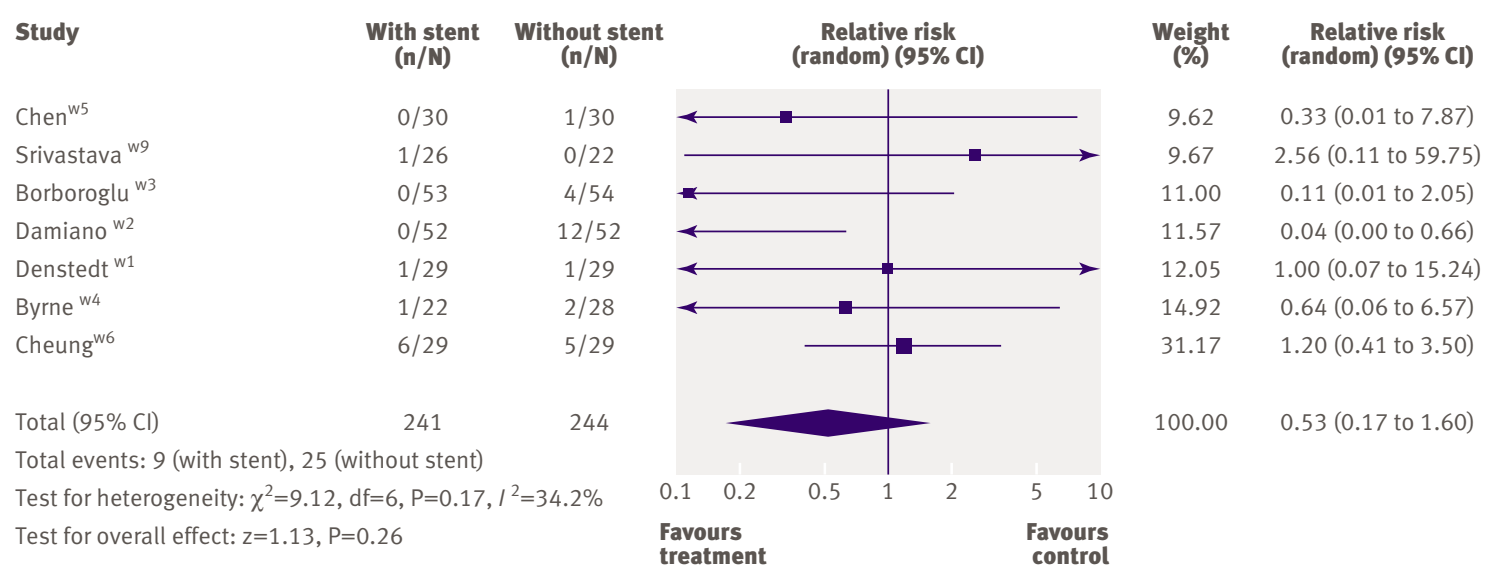




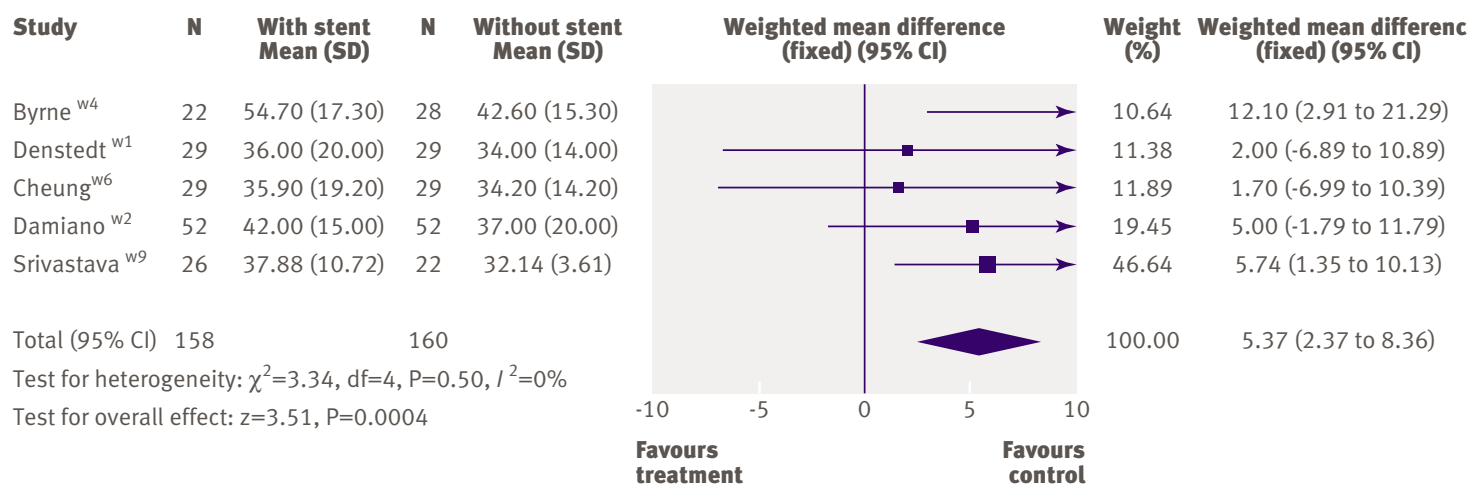

Fig $5 \mid$ Operation time (minutes) in patients with and without stents during ureteroscopy

al showed no difference in the impact on patients' quality of life between ureteric stents composed of firm or soft polymer. ${ }^{11}$ This trial, however, did not have a control group of participants without stents. In a non-randomised study, up to $80 \%$ of participants experienced urinary symptoms and pain associated with indwelling ureteric stents, which interfere with daily activities and result in a reduced quality of life. ${ }^{3}$ Chen et al reported return to normal physical activity in $80 \%$ of participants on the day after the procedures in both groups. $^{\text {w5 }}$

A few trials reported on cost implications. Not surprisingly, there was a higher cost associated with use of ureteric stents, but none of the trials reported on the cost effectiveness of this intervention.

\section{Limitations}

Lack of definition of uncomplicated ureteroscopy-Because of a lack of standardisation of the definition of "uncomplicated ureteroscopy," the decision not to insert a stent can be difficult. This was evident from the included trials. This decision is often affected by the technique, technology used, and experience of the operating surgeon. Denstedt et al defined uncomplicated ureteroscopy as no evidence of perforation or lack of clinically important oedema. ${ }^{\mathrm{w} 1}$ They did not, however, propose any objective criteria to assess the clinical importance of any oedema after the procedure but suggested that free flow of contrast into the bladder on retrograde pyelography should rule it out. Other studies used an endoscopic, non-validated grading of ureteric trauma and oedema on a scale of 0 (mild) to 2 (severe). ${ }^{2}$ w6

Performance and reporting of studies - There was a lack of standardisation of outcome measures, length of trial, and duration of stenting. The use of preprocedural antibiotics, trial design, stent material, patient population, assessment of health related quality of life, and cost-effectiveness data were all inconsistent. Studies reported pain scores as means with variance, although it is well recognised that data from visual analogue scales are often skewed and therefore may be more accurately analysed as medians. We were unable to access data from individual patients to assess whether comparison of medians rather than means may have altered our findings. Some trials allowed withdrawal after randomisation because of intraoperative complications, ${ }^{\mathrm{w} 2} \mathrm{w} 3$ leading to potential reporting bias. The longest follow-up was only six months. ${ }^{\text {w2 }}$

Quality of reporting - The general quality of trials was poor (table 2). Some participants were excluded from the analysis in a few trials because of intraoperative complications, making it difficult to assess the true effect of intervention. Most of the trials had small sample sizes.

\section{Implications for practice}

As stent placement after ureteroscopy seems to cause undesirable lower urinary tract symptoms maybe it should not be a standard practice. There are, however, many unanswered questions, and any recommendations would be potentially flawed because of a lack of standardisation of outcome measures, marked clinical heterogeneity, withdrawal after randomisation, imprecision in measurement of outcomes (large confidence intervals), and poor reporting of published clinical trials.

\section{Implications for research}

Research efforts should now be concentrated on higher quality, more rigorous randomised trials. As a minimum, these should use predefined ideally standardised measures of outcome and be multicentred to ensure that the studies give sufficiently precise estimate of the various outcomes. Trials should be protocol driven and a detailed protocol of how the project is to be conducted should be agreed before the start. The protocol should state the research objectives, reasons for the study, issues related to recruitment (inclusion and exclusion criteria), information to be collected at entry to the study, and interventions of interest, and there should be an agreed follow-up protocol. Outcome measures must include outcomes assessed by patients and ideally health economic outcome measures. The impact of variations in stent design, size, and material, and the effect of different types of intracorporeal lithotripsy sources on the requirement for stents need to be examined. 


\section{WHAT IS ALREADY KNOWN ON THIS TOPIC}

Stenting after ureteroscopy may lead to undesirable lower urinary tract symptoms with

limited benefits to patients

\section{WHAT THIS STUDY ADDS}

Stent placement after ureteroscopy results in considerable morbidity in the form of irritative lower urinary tract symptoms

It does not seem to influence stone free rate, rate of urinary tract infection, requirement for analgesia, or long term ureteric stricture formation

The role of stenting in uncomplicated ureteroscopy remains unclear

research unit is core funded by the Chief Scientist Office of the Scottish Executive Health Department.

Contributors: GN, SMCC, and JN'D contributed to the idea and designed the review protocol. GN and SMcC undertook screening of articles, data extraction, and quality assessment. JN'D contributed to the drafting and critical revision of the report. JC performed the statistical analyses. All authors contributed to drafting the manuscript and approved the final version. GN is guarantor.

Funding: None.

Competing interests: None declared.

Ethical approval: Not required.

1 Gettman MT, Segura JW. Management of ureteric stones: issues and controversies. BJU Int 2005;95(suppl 2):85-93.
2 Ryan PC, Lennon GM, McLean PA, Fitzpatrick JM. The effects of acute and chronic JJ stent placement on upper urinary tract motility and calculus transit. Br J Urol 1994;74:434-9.

3 Joshi HB, Newns N, Stainthorpe A, MacDonagh RP, Keeley FX Jr, Timoney AG. Ureteral stent symptom questionnaire: development and validation of a multidimensional quality of life measure. J Urol 2003;169:1060-4.

4 Duvdevani M, Chew BH, Denstedt JD. Minimizing symptoms in patients with ureteric stents. Curr Opin Urol 2006;16:77-82.

5 Singh I, Gupta NP, Hemal AK, Aron M, Seth A, Dogra PN. Severely encrusted polyurethane ureteral stents: management and analysis of potential risk factors. Urology 2001;58:526-31.

6 Willis NS MR, Craig JC. Renal group. Cochrane library. Issue 4. Chichester: Wiley, 2003.

7 Higgins JP, Thompson SG, Deeks JJ, Altman DG. Measuring inconsistency in meta-analyses. BMJ 2003;327:557-60.

8 Ali W A-DM, Al-Bareeq R, Samiei MR, Al-Mutawa S. The evaluation of not stenting after uncomplicated ureteroscopy: a randomized prospective study. Bahrain Medical Bulletin 2004;26:3-5.

9 Boddy SA, Nimmon CC, Jones S, Ramsay JW, Britton KE, Levison DA, et al. Acute ureteric dilatation for ureteroscopy. An experimental study. Br J Urol 1988;61:27-31.

10 Teichman JM, Vassar GJ, Bishoff JT, Bellman GC. Holmium:YAG lithotripsy yields smaller fragments than lithoclast, pulsed dye laser or electrohydraulic lithotripsy. J Urol 1998;159:17-23.

11 Joshi HB, Chitale SV, Nagarajan M, Ivving SO, Browning AJ, Biyani CS, et al. A prospective randomized single-blind comparison of ureteral stents composed of firm and soft polymer. J Urol 2005;174:2303-6.

Accepted: 10 January 2007 